\title{
Variantes y reelaboración del exemplum del hombre y el ave en la Disciplina Clericalis, Barlaam y Josafat y el Libro del caballero Zifar
}

\section{Variants and reconfiguration of man and bird exemplum in Disciplina Clericalis, Barlaam y Josafat, and the Libro del caballero Zifar}

Sulemi Bermúdez Callejas

El Colegio de México

En la tradición medieval, era común el uso de exempla como un método de enseñanza de la época. El exemplum del hombre y el ave aparece en varias obras medievales a veces como parte de antologías de exempla y otras como núcleo temático e ideológico de la narración. En este artículo, se analiza el motivo de los tres consejos de un pájaro a un cazador y su reelaboración en el Barlaam y Josafat, la Disciplina Clericalis y el Libro del caballero Zifar. En estos tres relatos se presentan cambios en el orden de los consejos como en elementos indiciales (el lugar, el ave, el hombre, el tesoro). Las variaciones responden tanto a la mezcla de tradiciones como a la intención autoral.

Palabras Clave: variación, motivo, ave, cazador, exempla, Barlaam, Disciplina, Zifar

In medieval tradition, the use of exempla was a common educational method. The exemplum of the man and the bird appears in several medieval works, at times as part of anthologies and at others as a thematic and ideological core of the narrative. This article analyses the motif of three counsels a bird gives to a man, and its reconfiguration in the Barlaam y Josafat, the Disciplina clericalis and the Libro del caballero Zifar. In these three tales there are changes to the order of the counsels and the main elements - the location, the bird, the man and the treasure. The variations are due to the mixture of traditions and the author's intention.

KeYwORDs: variation, motif, bird, hunter, exempla, Barlaam, Disciplina, Zifar

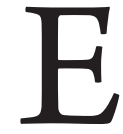

n la tradición medieval, la originalidad no era un fin que se persiguiera, por lo que era común que algunas historias folclóricas pasaran de una obra a otra. Juan Manuel Cacho Blecua afirma que los autores medievales no valoran la inventio como lo hacemos hoy en día, sino que "pretenden 
insertarse en una tradición de la que imitan algunos de sus más prestigiados arquetipos (imitatio) que repiten cansinamente hasta la saciedad, en múltiples ocasiones introduciendo algunas variantes" (Cacho Blecua, "Introducción al estudio", 34). Esto sucede con algunos exempla que subsistieron durante siglos con ciertas variantes.

Según María Jesús Lacarra, los exempla eran relatos que gozaban de gran popularidad porque presentaban modelos de conducta aceptables de forma amena. Estas historias tenían un objetivo doctrinario y se basaban en el docere et delectare (instruir y deleitar), principio que regía las actividades intelectuales de la época ("El cuento en la Edad Media", 10). La autora señala que el ejemplo tenía una función didáctica a partir de analogías y del método inductivo. Un exemplum que tuvo una amplia difusión en la Edad Media fue el del hombre y el ave. Esta historia aparece en varias obras, entre ellas en el Barlaam y Josafat, en la Disciplina Clericalis, el Libro de los exemplos por a.b.c., La vida y fábulas de Ysopo, la Gesta Romanorum, el Isopete e incluso en el Libro del caballero Zifar. En este trabajo, estudio la reelaboración y las variantes de este exemplum en el Barlaam, la Disciplina y el Zifar que volvieron significativo el motivo de los tres consejos de un ave en la tradición medieval.

Para este análisis considero una definición amplia de motivo que integra las propuestas de Aurelio González y Stith Thompson. El primer autor define motivo como la unidad mínima de la narración que expresa y conserva su significado en un nivel más profundo de la fábula dentro su propia cadena sintagmática ("El concepto de motivo", 381). Thompson considera que el motivo folclórico es "el elemento más pequeño en un cuento que tiene el poder de persistir en la tradición” (El cuento folklórico, 528). A partir de estas dos definiciones, me parece que el exemplum del hombre y el ave correspondería a un tipo que conjuga varios motivos: personajes, objetos, acciones, según la clasificación de Thompson. Ahora bien, en este análisis busco la función de los motivos dentro de la obra, pues según Cacho Blecua es necesario tener en cuenta "su situación en la intriga en la que se insertan en conexión con una tradición que ratifican, renuevan o crean" ("Introducción al estudio", 50).

El motivo de los tres consejos que le da un pájaro a un cazador (K 604) proviene de la tradición oriental, específicamente del folclor búdico (Disciplina clericalis, n. 32, 238). Llegó a la literatura hispánica a través de la Disciplina clericalis (s. XII) y del Barlaam y Josafat (s. XIII), ambos escritos originalmente en latín. La Disciplina es una compilación de cuentos orientales reunida por Pedro Alfonso, judío converso cuyo saber enciclopédico se ocupaba de las siete artes liberales. Este libro didáctico tenía por objetivo formar a un individuo perfecto. Lacarra asegura que una de las fuentes de Pedro Alfonso fue 
el Barlaam y Josafat, aunque todavía queda en duda si el autor tradujo al latín un texto preexistente escrito en alguna lengua oriental o si fue el compilador directo de varios textos ("Introducción”, 23). El exemplum del rústico y la avecilla aparece en el segundo núcleo temático dedicado a la relación del hombre con sus semejantes y ocupa el lugar número XXII dentro de la compilación. En esta versión, un hombre que descansaba en su hermoso vergel caza a una avecilla que lo convence de dejarla en libertad a cambio de tres "maneras de sabiduría”. El hombre decide soltarla y la avecilla le da tres consejos, que después ella misma pondrá a prueba.

El primer consejo marca la pauta de los siguientes, ya que el ave señala los peligros que supone el exceso de credulidad como principio básico de comportamiento. Pareciera una instrucción hecha a la medida de los educandos, en donde se pone en duda todo aquello que se oye y cuyo objetivo es formar un carácter juicioso, en este caso del rústico $y$, en consecuencia, de los lectores y oyentes del exemplum. Posteriormente, se resalta el carácter efímero de las pertenencias y su cuidado con un par de consejos: por un lado, la avecilla le aconseja al rústico conservar lo que le pertenece; $y$, por otro, le pide no lamentarse por lo perdido; es decir, sólo podrá preservar aquello que verdaderamente posee. Acerca de otras posesiones no es necesario condolerse, pues no eran suyas en realidad.

Un giro muy peculiar de esta versión es el epílogo del exemplum titulado "De la credibilidad de los libros", donde los primeros consejos de la avecilla ("No creas en todo lo que se te diga" y "Lo que fuere tuyo, consérvalo siempre”) se reelaboran en dos historias muy breves. De tal manera que la estructura de caja china se extiende dentro del mismo exemplum, pues se cuentan dos historias de personajes que pareciera que lo leyeron o escucharon. En la primera, un filósofo aconseja a su hijo leer todo lo que encuentre, pero no creer en todo lo que lee. El joven asiente y refuerza la idea con una metáfora arbórea donde compara a los libros con árboles y a los conocimientos con frutos: "muchos son los árboles, pero no todos dan fruto; muchos dan frutos, pero no todos comestibles", sentencia que rectifica el consejo del ave. Discriminar el consejo bueno del malo era tan importante como discernir entre los buenos y los malos libros. La reflexión acerca de la lectura que propone Pedro Alfonso valida el saber de su propia obra, dado que la Disciplina es fundamentalmente un libro de consejos para alcanzar la grandeza de espíritu. Con esta sentencia, Pedro Alfonso se distancia de los libros perniciosos y pone en relieve aquellos que pueden brindar una buena orientación, como la Disciplina misma. En la otra historia, únicamente aparece la advertencia que hace un árabe a su hijo: "Hijo mío, no dejes lo presente por lo futuro, no vayas a perder 
uno y otro, como le pasó al lobo con los bueyes que le prometiera un aldeano" (Alfonso, Disciplina clericalis, 81). Este consejo remite al segundo del avecilla, pero tiene como intertexto la fábula de Esopo "El labrador, el lobo, la raposa y el queso", en la que el labrador no cumple la palabra de darle sus bueyes al lobo. Ambos tratan de arreglar este altercado y recurren a la raposa como juez. Sin embargo, la raposa con su astucia logra engañar al lobo al decirle que en lugar de los bueyes tendrá un gran queso, que sólo es el reflejo de la luna dentro de un pozo. La moraleja de esta historia concuerda con los tres consejos dados por el ave. El breve consejo del árabe tan sólo sirve para dar pie al siguiente exemplum de la Disciplina, que precisamente hace referencia a la historia antes descrita: "Ejemplo del labrador y del lobo y del juicio de la zorra".

El exemplum del rústico y el ave también aparece en Barlaam y Josafat bajo el título de "El pajarero y el ruiseñor". El Barlaam es un texto de origen oriental, cuyo argumento se retoma de la leyenda cristiana del príncipe Josafat y su maestro Barlaam. Esta es una versión de una historia del siglo VI a.C. que contaba la conversión del príncipe indio Siddharta Gautama en un 'buda' o 'iluminado' (Lacarra, "El cuento en la Edad Media", 23). Lacarra señala que hay varios paralelismos entre esta obra y el Sendebar; por ejemplo, a nivel estructural ambas se construyen a partir de un marco narrativo en el que se insertan cuentos y parábolas a manera de exemplum. La investigadora subraya que "a partir del siglo XII no habrá ejemplario latino donde no se incluya alguno de sus conocidísimos cuentos como 'La trompeta de la Muerte', III, 1, 'Las dos arcas', III, 2, o 'El cazador y el ruiseñor', VII, 5. Con el Barlaam estamos ante la más temprana adaptación de la narrativa oriental al Occidente cristiano" ("El cuento en la Edad Media", 25). La historia de "El pajarero y el ruiseñor" se encuentra en el capítulo X de esta obra, dentro del marco narrativo que sirve para ilustrar una enseñanza de Barlaam a Josafat. El joven se encuentra apesadumbrado porque le gustaría escapar de los castigos reservados a los pecadores y merecer la alegría de los justos. En esta historia, el adoctrinamiento religioso trasciende el docere et delectare al igual que en la Disciplina. Lacarra explica que "frente a la rapidez con la que los narradores extraían la lección moral en el Calila y en el Sendebar, el sabio Barlaam se detiene en explicar la adecuación entre la historia y el discurso religioso; los elementos de la anécdota son sometidos a un proceso exegético, mostrando sus correspondencias alegóricas" ("El cuento en la Edad Media", 25); así el maestro cumple también con el papel de exégeta por lo que sus relatos tendrán un adoctrinamiento religioso.

En este pasaje del Barlaam, el maestro recurre al ejemplo del pajarero y el ruiseñor que le había contado "un hombre muy sabio" para evidenciar la insensatez de los idólatras. La referencia a la anterioridad del relato tiene 
una función doble, pues valida el discurso por la sabiduría del emisor y también cumple la función de una marca de oralidad. Hay que recordar que el Barlaam fue traducido al latín hasta el siglo XI, pero la tradición oriental de la que abreva es remota. Con una estructura de caja china, Barlaam introduce tal ejemplo para aleccionar a Josafat sobre los idólatras, a quien compara con el pajarero de la historia. En esta versión, los consejos cambian de orden: el primer consejo que da el ruiseñor al pajarero es que no intente alcanzar lo inalcanzable; el segundo, se refiere a no lamentarse por el pasado y, por último, el ruiseñor persuade al pajarero para que desconfíe de lo inverosímil. Al cambiar el orden de los consejos, se modifica la prioridad de las enseñanzas, pues es frecuente que la primera de ellas se tome como la primordial. Aunque en este caso resulta un tanto extraño que al ser un exemplum para apartar a Josafat de la idolatría no se haya priorizado desconfiar de lo improbable para combatir el paganismo.

Según Wagner, el ejemplo de "El pajarero y el ruiseñor" del Barlaam pasó al Libro del caballero Zifar (Wagner, El libro del cauallero Zifar, apud Cacho Blecua, "Del 'exemplum', 221). No obstante, este punto es debatible, pues para Juan Manuel Cacho Blecua es casi imposible asegurar la transmisión de este libro al otro, dado que muchos exempla se memorizaban en las escuelas y eran parte de la didáctica medieval. Además, aún no se sabe si el autor del Zifar tuvo acceso a los manuscritos de este texto ("Del 'exemplum', 221). El tema principal del Zifar son las enseñanzas del Rey de Mentón, encaminadas a conseguir la gracia de Dios, cuestión que lo acerca al Barlaam. Su concepción estructural podría responder al deseo de conservar cierta unidad narrativa donde intercala otro tipo de materiales en el marco narrativo base. De tal manera que el autor del Zifar, según Cacho Blecua, "engarzó y remodeló diferentes tradiciones genéricas, modificando y renovando así el panorama literario anterior" ("Del 'exemplum', 209). La técnica narrativa de cajas chinas o mise en abyme se introduce en un relato de largo aliento, lo que marca una renovación de la tradición literaria, pues si bien utiliza los recursos literarios propios de la época, también plantea una historia más compleja.

Dentro del marco narrativo de los Castigos del rey de Mentón, el exemplum del cazador y la calandria corresponde al nivel narrativo 4, según Carmen Hernández Valcárcel, pues está "puesto en boca del médico para ilustrar gráficamente su consejo" ("Algunos aspectos del cuento", 477), que sería el nivel 3. Cabe decir que la manera de enseñar en el Zifar también seguía la pauta del docere et delectare, que en este texto se anuncia como "educar mediante el solaz", de ahí que la inserción de pequeños cuentos tenga sentido dentro de un marco más amplio. Según Xiomara Luna, en esta sección 
se formula "una didáctica de la palabra, expresión de la mesura y del 'buen seso' que [...] guía la construcción de los personajes y constituye uno de los aspectos ideológicos fundamentales del libro" ("El episodio cómico", 129). Este exemplum educa "mediante el solaz" para discernir entre el buen y el mal consejo. Es revelador que una de las primeras enseñanzas de la segunda parte del Zifar sea precisamente sobre el carácter de los consejos. Pareciera que este discurso se torna autorreferencial y busca aleccionar al lector sobre la eficacia de los consejos vertidos en el libro al afirmar su calidad, como sucede en la Disciplina. Al validar el discurso por medio de un ejemplo folclórico la novela se reviste de un carácter formal.

En el Zifar hay una ampliación de la historia del exemplum, pues el fin del cuento no se da cuando el ave corrobora sus enseñanzas, como en el Bar50 laam y la Disciplina, sino que concluye con el motivo K 1041.1 del índice de Thompson, es decir, con el "intento de vuelo con plumas de ave". El cazador acude con un trasechador que lo incita a pegarse plumas para intentar volar. Esta escena sólo refuerza el carácter de los consejos del ave que cobra fuerza por la fatalidad del desenlace. Este motivo posiblemente es una apropiación del mito de Ícaro. Cacho Blecua sugiere que esta ampliación de la trama surge de una frase del Barlaam: "y tú tratas de capturarme cuando no puedes alcanzarme en el aire" (Baarlam, 71). ${ }^{1}$ El investigador contrasta su teoría con la de Rameline Marsane, quien atribuye la amplificación a la frase "Et quando potes lapidemhabere de me volante?" de la Disciplina. Ahora bien, Cacho Blecua localiza otras dos posibles fuentes: por un lado, las Metamorfosis de Ovidio, que se memorizaba en las escuelas y se conocían en el ámbito clerical; y, por otro, la General estoria de Alfonso X, libro en el que se retoma el mito de Ícaro y Dédalo. El estudioso encuentra coincidencias significativas entre el relato alfonsí y el exemplum del Zifar, pues este último retoma elementos que aparecen en el primero, ya que el cazador, en una muestra de desobediencia a los consejos, se pega plumas con cera y se tira desde una torre; mismo caso del Ícaro alfonsí. Cacho Blecua apunta que

el Zifar sería inexplicable sin la experiencia artística que supuso la prosa surgida en los talleres del monarca castellano, quien había recalcado el valor ejemplar de la historia, había integrado los más diversos géneros, desde la exégesis bíblica a la hagiografía, y había contribuido a la incorporación y difusión de los más variados materiales procedentes de la tradición grecolatina y del mundo

1 El autor cita estas dos versiones de la frase: "E tu non puedes bolar por el ayre commo yo" (Baarlam, P, 93, 1745-1746); "E tentaste tomarme commo non puedas yr por la mi carrera” (Baarlam, S, 381, 984-985), (“Del 'exemplum”, 222). 
árabe, consiguiendo unos logros artísticos y unos horizontes narrativos desconocidos en romance hasta entonces ("Del 'exemplum", 223).

La mezcla de fuentes árabes y grecolatinas en un mismo exemplum muestra la confluencia de diversas tradiciones que había en la península ibérica en la Edad Media. Es importante mencionar que el Zifar se considera la primera novela autóctona. El afán del autor del Zifar de ficcionalizar un relato folclórico usando diversas técnicas, en este caso la amplificatio, comprueba la necesidad de renovación de los modelos literarios. Luna plantea que este exemplum entraña uno de los núcleos temáticos e ideológicos fundamentales del Zifar. Este episodio es cómico porque la enseñanza se consigue por medio del escarnio, ya que el ingenuo y el necio que se dejaban engañar causaban risa ("El episodio cómico", 6). En el Barlaam y la Disciplina, el episodio carece de este matiz cómico porque su función didáctica tiene un tono más severo. Los consejos del ave se corroboran en la misma interacción de los dos personajes, mientras que en el Zifar hay una escisión temporal, pues hay una acción del cazador posterior al diálogo con la calandria.

También hay otras variantes en los consejos que aparecen en las tres versiones del exemplum. Por ejemplo, el orden en que el ave brinda los consejos no es el mismo. Como se dijo arriba, el orden de la enunciación no es fortuito: responde a una serie de procedimientos narrativos internos del relato o a la valoración del autor para enfatizar la relevancia de cada consejo. Quizá esto podría explicarse como lo hace Cacho Blecua respecto al Zifar, pues señala que "el cambio de orden se explica por la acomodación de la materia narrativa al nuevo contexto" ("Del 'exemplum", 228). En el Zifar y en la Disciplina, el primer consejo ("No creas en todo lo que se te diga") es el último en el Barlaam. Mientras que el segundo consejo ("No te lamentes por el pasado") del Barlaam y del Zifar es el tercero de la Disciplina. El primer consejo del Barlaam también es el primero del Zifar ("No intentes alcanzar lo inalcanzable"), mismo que no aparece en la Disciplina, donde se recurre a un nuevo consejo ("Lo que tuyo fuere, consérvalo siempre"), como se puede apreciar en el cuadro 1 . En los tres relatos se hace hincapié en la desconfianza ante lo inverosímil como principio lógico del buen entendimiento. También en las tres obras prevalece el consejo que dicta la resignación ante los bienes perdidos, punto nodal del cristianismo. Los matices que aparecen entre una versión y otra dan cuenta de las posibilidades de reelaboración de un mismo exemplum, ya que el autor puede cambiar el sentido de la frase tanto por la traducción del latín a lengua romance, como por la estructura del texto o la intención de los consejos. 
No sólo en los consejos se presentan variantes, también las hay en los elementos indiciales como en la descripción del lugar, el ave, el tesoro e incluso en la forma de nombrar al receptor de los consejos (cuadro 2). Sobre este último, cabe destacar que, en los ejemplarios, como la Disciplina o el Libro de los exenplos, se denomina al personaje simplemente como "hombre". La escueta caracterización se debe a un distanciamiento en el mismo relato, debido a que no pertenece a una estructura más compleja. Así pueden omitirse detalles para lograr mayor universalidad. Aunque hay una inconsistencia en la Disciplina, pues se le denomina como "rústico" en el título del exemplum —mismo caso que en La vida y fábulas del Ysopo-, aunque esta denominación no se repetirá en la narración. La precisión en el título dota al personaje de una característica particular: no es cualquier hombre, es un campesino que cuida un vergel. En el Zifar y en el Barlaam, se caracteriza al personaje con mayor detalle, ya que su aparición tendrá sentido en los marcos en que se inscribe. El pajarero y el cazador no son hombres que por azar atrapan aves, son personajes que tienen la intención explícita de ir en su búsqueda. De ahí que sea todavía más significativo que suelten a su presa una vez que la han atrapado y, más aún, que le devuelvan su libertad a cambio de consejos.

De igual manera, hay variaciones en la descripción del lugar que reflejan cambios estructurales. Mientras que en el Barlaam la descripción del espacio se omite, en la Disciplina, Pedro Alfonso describe el lugar con amplitud: "Un vergel en donde - pues corrían varios riachuelos - había hierba muy verde, y por la amenidad del lugar acudían a él avecillas que cantaban a coro con la varia modulación de sus trinos" (Disciplina, 80). Este locus amoenus remite a un lugar de paz que recuerda al jardín del Edén. En La vida y fábulas del Ysopo también se refiere ampliamente el espacio: "Tenía un aldeano una huerta con sus fuentes corrientes limpias e muy ornadas de yervas y flores, por que muchas vezes venían allí las aves" (La vida y fábulas del Ysopo, f. LXVr). El Libro de los exenplos sigue con este tipo de descripción, aunque de una manera más sencilla: "Dizen que um ombre tenia un vergel en que coria agua e tenia yerba verde..." (Libro de los exemplos por a.b.c, 110). Por último, en el Zifar, más que una descripción del lugar hay una puesta en escena: "Dize el cuento que vn caçador fue a caça con sus redes...” (Zifar, 264). Al conjugar la descripción del personaje y del lugar se puede inferir que el espacio ocupa un lugar de mayor significación en los ejemplarios donde se omite la caracterización del personaje, como en la Disciplina, el Libro de los exenplos y La vida y fábulas del Ysopo; mientras que en el Zifar y el Barlaam, los personajes se describen de acuerdo con su oficio (cazador y pajarero), por lo que la descripción del lugar se obvia en función del espacio donde habitualmente realizan sus actividades estos personajes. 
Otra cuestión que resulta significativa es la caracterización del ave en algunos relatos. Carmen Elena Armijo señala que "el vuelo predispone a los pájaros para ser símbolos de las relaciones entre cielo y tierra. Aún más generalmente, las aves simbolizan los estados espirituales, los ángeles y los estados superiores" ("Reelaboración y simbolismos", 235). En la Disciplina se presenta con el nombre genérico "avecilla”. De nuevo, Pedro Alfonso prefiere una denominación general, quizá para que el ejemplo pueda ser interpretado libremente por el lector. También bajo ese mote aparece en La vida y fábulas del Ysopo. En el Libro de los exenplos y en el Barlaam, es un ruiseñor el que aconseja al personaje. Mientras que en el Zifar esta ave toma la forma de calandria. Estas aves no tenían el mismo valor simbólico en el imaginario medieval (Malaxecheverría, Bestiario medieval, 115-119 y Charbonneau-Lassay, Bestiario de Cristo, 431-437). La calandria era una representación de Cristo y el ruiseñor de la diosa del Luscinia, epíteto de Hera ("la que trae la luz"), según Isidoro de Sevilla (Etimologías). Cacho Blecua aclara el tránsito de ruiseñor a calandria:

La identificación de ambas aves en la tradición lexicográfica hispana queda atestiguada en el Vocabulario de romance en latín de Nebrija (1981), quien define la alondra (calandria) con las voces latinas "luscinia - ae. Luscioniala -ae" mientras que el ruiseñor es también "luscinia -ae. Lusciola -ae. Philomena -ae" (“Del 'exemplum'”, 228).

La similitud de la alusión latina para las dos aves explica la ambivalencia en las traducciones. Es posible que el autor del Zifar haya tomado el ejemplo directamente del latín y se haya "perdido" en la traducción. Sin embargo, la calandria tendría un significado más profundo por su relación con lo sagrado, ya que se le asociaba con Cristo y, por lo tanto, estaba prohibido comerla (Charbonneau-Lassay, Bestiario de Cristo, 435). Tanto en el Barlaam como en el Libro de los exenplos, se presenta al ruiseñor como un ave asociada a la luz, que podría ser la luz del conocimiento.

Hay otra variante respecto al tesoro que el ave dice llevar en el vientre. En este caso, la descripción más general es la del Zifar, pues sólo se dice que tiene una piedra preciosa sin definir cuál es; y lo único que se aclara es su tamaño: "tan grande commo vn hueuo de estrus". Esta comparación es la misma del Barlaam, aunque aquí se especifica que se trata de una perla, que en el cristianismo se equipara con la sabiduría proveniente de las enseñanzas de Jesucristo. En el Libro de los exenplos y en La vida y fábulas del Ysopo, el tesoro tampoco se ciñe a una clasificación específica, ya que "jacinto” podía 
referir por igual al circón, al rubí, al topacio y a un tipo de cuarzo de color rojo. Aunque también esta piedra tiene una significación en el cristianismo, pues representa al undécimo cimiento del muro de la ciudad de Dios (Apocalipsis, 21:20). En estas dos obras se determina la magnitud de la piedra al decir que era del "pesso de una honça". Esta medida es la misma de la amatista de la Disciplina. Cabe decir que durante la Edad Media la amatista era valuada por encima de los diamantes. La exageración del tamaño del tesoro guarda relación con el consejo del ave acerca de desconfiar de lo inverosímil, pues sería imposible que un ave tuviera una piedra de tales dimensiones en su vientre. La elección de una piedra u otra guarda una relación estrecha con aquello que significaba en el imaginario medieval.

A manera de conclusión, hay que señalar que la aparición de los exem54 pla en estas obras responde al estilo narrativo de la época que tenía como finalidad enseñar mientras se deleitaba al lector u oyente. En la Disciplina y el Libro de los exenplos, las descripciones suelen ser más sobrias, ya que sólo eran recopilaciones de exempla, por lo que las cuestiones de estilo se dejan de lado casi por completo. No sucede de esta forma en el Barlaam que retoma el exemplum para ejemplificar un modo de actuar conforme a la doctrina religiosa. En el Zifar, la reelaboración del ejemplo incluso da lugar a una amplificatio que recupera un motivo de la tradición grecolatina por medio de un texto alfonsí. De manera que el exemplum se renueva al entrar en contacto con estructuras ficcionales muy cercanas a las estrategias narrativas de la novela moderna.

Las variantes en los consejos y en los elementos indiciales son significativas, puesto que delimitan el relato, ya sea al centrar la atención en alguna de las enseñanzas o al incluir descripciones o especificaciones de los personajes y el espacio. Los símbolos intrínsecos que guardan las aves (calandria y ruiseñor) o las piedras preciosas (jacinto, perla, amatista) fortalecen la estructura de la historia. Así, aunque la originalidad no se persiga como un fin en sí mismo, se presenta debido a estas variantes, pues con la reelaboración del ejemplo los autores encuentran la esencia del cuento folclórico y la transforman según la estructura de cada marco narrativo. 
Cuadro 1. Consejos del ave

\begin{tabular}{|l|l|l|l|}
\hline \multicolumn{1}{|c|}{ Obra } & \multicolumn{1}{|c|}{ Primer consejo } & \multicolumn{1}{c|}{ Segundo consejo } & \multicolumn{1}{c|}{ Tercer consejo } \\
\hline $\begin{array}{l}\text { Barlaam } \\
\text { y Josafat }\end{array}$ & $\begin{array}{l}\text { A) Nunca intentes } \\
\text { alcanzar lo inalcan- } \\
\text { zable }\end{array}$ & $\begin{array}{l}\text { B) No te lamentes } \\
\text { por algo pasado }\end{array}$ & $\begin{array}{l}\text { C) No te fíes jamás } \\
\text { de lo inverosímil }\end{array}$ \\
\hline $\begin{array}{l}\text { Disciplina } \\
\text { clericalis }\end{array}$ & $\begin{array}{l}\text { C) No creas en todo } \\
\text { lo que se te diga }\end{array}$ & $\begin{array}{l}\text { D) Lo que tuyo fuere, } \\
\text { consérvalo siempre }\end{array}$ & $\begin{array}{l}\text { B) No te lamentes } \\
\text { por lo que hayas per- } \\
\text { dido }\end{array}$ \\
\hline $\begin{array}{l}\text { Libro del ca- } \\
\text { ballero Zifar }\end{array}$ & $\begin{array}{l}\text { C) Non creas a nin- } \\
\text { guno aquello que } \\
\text { vieres e entendieres } \\
\text { que non puede ser }\end{array}$ & $\begin{array}{l}\text { B) Non trabajes en } \\
\text { pos la cosa perdida, } \\
\text { sy que la non pue- } \\
\text { das cobrar }\end{array}$ & $\begin{array}{l}\text { A) Non acometas } \\
\text { cosa entiendas } \\
\text { que nuedes aca- } \\
\text { bar }\end{array}$ \\
\hline $\begin{array}{l}\text { Libro de los } \\
\text { exenplos }\end{array}$ & $\begin{array}{l}\text { C) Non creas todo } \\
\text { lo que te dixieren }\end{array}$ & $\begin{array}{l}\text { D) Lo que tuyo fue- } \\
\text { re siempre lo guar- } \\
\text { da e lo ten }\end{array}$ & $\begin{array}{l}\text { B) Por cossa que } \\
\text { pierdas nunca ayas } \\
\text { dolor }\end{array}$ \\
\hline $\begin{array}{l}\text { La vida } y \\
\text { fábulas del } \\
\text { Ysopo }\end{array}$ & $\begin{array}{l}\text { C) No creas a todas } \\
\text { las palabras que oye- } \\
\text { res, señaladamente } \\
\text { a aquéllas que no } \\
\text { parecen verdaderas }\end{array}$ & $\begin{array}{l}\text { D) Guarda lo que es } \\
\text { tuyo }\end{array}$ & $\begin{array}{l}\text { B) No te duelas de } \\
\text { las cosas perdidas, } \\
\text { las quales no puedes } \\
\text { recobrar }\end{array}$ \\
\hline
\end{tabular}

Cuadro 2. Consejos del ave

\begin{tabular}{|l|l|l|l|l|}
\hline \multicolumn{1}{|c|}{ Obra } & Personaje & Ave & \multicolumn{1}{|c|}{ Lugar } & \multicolumn{1}{c|}{ Tesoro } \\
\hline $\begin{array}{l}\text { Barlaam } \\
\text { Josafat }\end{array}$ & Pajarero & Ruiseñor & No se describe. & $\begin{array}{l}\text { Una perla más } \\
\text { grande que un } \\
\text { huevo de aves- } \\
\text { truz }\end{array}$ \\
\hline $\begin{array}{l}\text { Disciplina } \\
\text { clericalis }\end{array}$ & $\begin{array}{l}\text { Hombre / } \\
\text { rústico }\end{array}$ & Avecilla & $\begin{array}{l}\text { Un vergel en donde } \\
\text {-pues corrían varios } \\
\text { riachuelos- había hier- } \\
\text { ba muy verde, y por la } \\
\text { amenidad del lugar acu- } \\
\text { dían a él avecillas que } \\
\text { cantaban a coro con la } \\
\text { varia modulación de sus } \\
\text { trinos. }\end{array}$ & \\
\hline
\end{tabular}




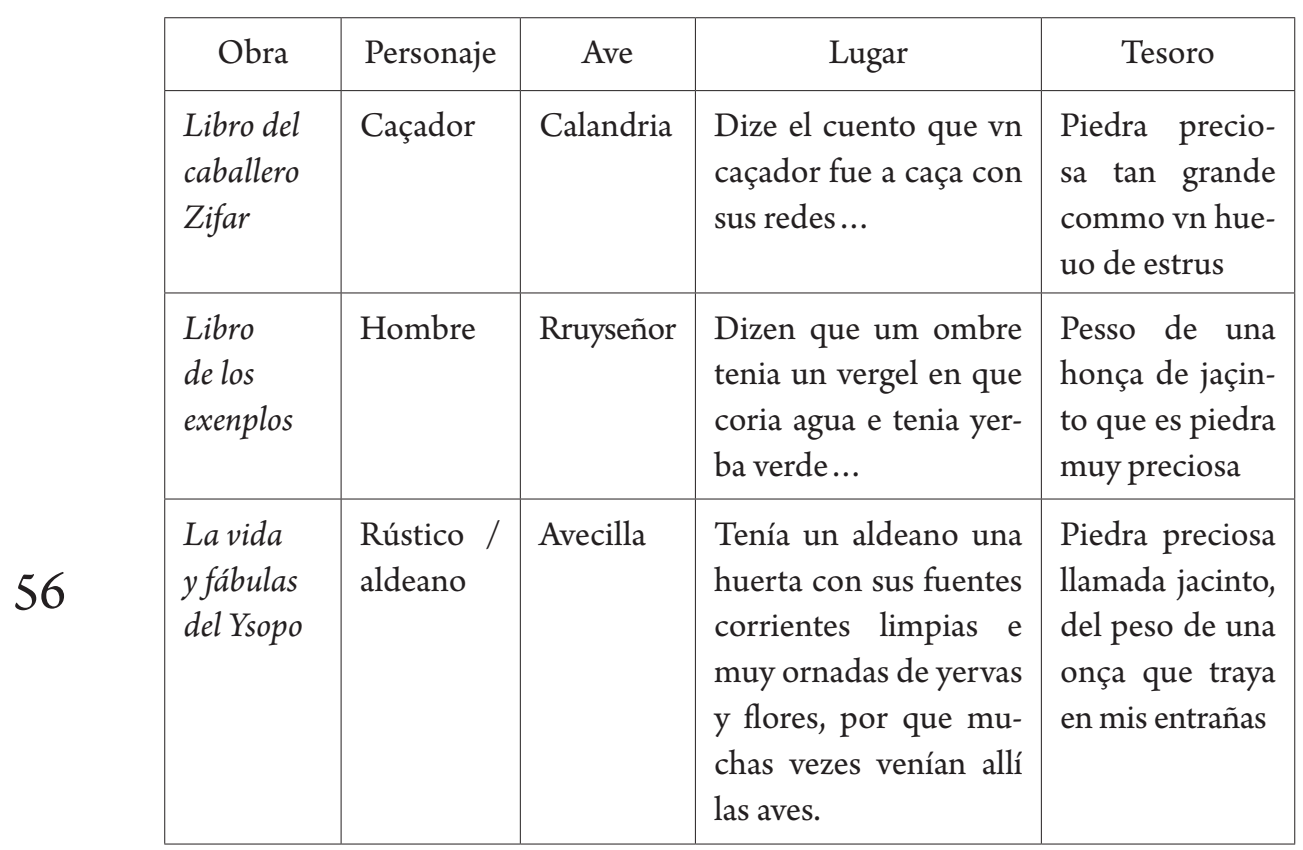

\section{BiBLIOGRAFÍA}

Alfonso, Pedro, Disciplina clericalis, introd. y notas de María Jesús Lacarra, trad. de Esperanza Ducay, Zaragoza: Guara, 1980.

Armijo, Carmen Elena, "Reelaboración y simbolismos en el 'enxienplo del caçador e la calandria' en el Libro del caballero Zifar”, en Karla Xiomara Luna Mariscal, Axayácatl Campos y Aurelio González (eds.), Zifar y sus libros: 500 años, México: El Colegio de México, 2015, 231-240.

Barlaam y Josafat. Redacción bizantina anónima, ed. de Pedro Bádenas de la Peña, Madrid: Siruela, 1993.

Cacho Blecua, Juan Manuel, "Del 'exemplum' a la 'estoria fictícia': la primera lección de Zifar”, en Juan Salvador Paredes Núñez (coord.), Tipología de las formas narrativas breves románicas medievales, Granada: Universidad de Granada, 1998, 209-236.

Cacho Blecua, Juan Manuel, "Introducción al estudio de los motivos en los libros de caballerías: la memoria de Román Ramírez”, en Eva Belén Carro, Laura Puerto y María Sánchez (eds.), Libros de caballerías (del Amadís al Quijote), Salamanca: Seminario de Estudios Medievales y Renacentistas, 2002, 27-53. 
Charbonneau-Lassay, Louis, Bestiario de Cristo, Palma de Mallorca: Olañeta, 1997.

GonZÁlez, Aurelio, "El concepto de motivo: unidad narrativa en el Romancero y otros textos tradicionales", en Lillian von der Walde Moheno (ed.), Propuestas teórico-metodológicas para el estudio de la literatura hispánica medieval, México: Universidad Nacional Autónoma de México -Universidad Autónoma Metropolitana, 2003, 353-384.

Hernández Valcárcel, Carmen, "Algunos aspectos del cuento en el Libro del caballero Zifar: estructuras de la narrativa breve”, en María Isabel Toro Pascua (ed.), Actas del III Congreso de la Asociación Hispánica de Literatura Medieval, Salamanca: Biblioteca Española del Siglo XV, 1994., t. 1, 469-478.

Isidoro de SEvilla, Etimologías, ed. de José Oroz Reta y Manuel A. Marcos Casquero, Madrid: Católica, 1983.

Lacarra, María Jesús, "El cuento en la Edad Media”, en Cuentos en la Edad Media, Madrid: Castalia, 1986, 9-85.

La vida y fábulas del Ysopo, Valencia, 1520, ed. de Diego Romero Lucas, Revista Lemir, 5, 2001.

Libro de los exemplos por a.b.c., ed. y prol. de John Esten Keller, Madrid: Consejo Superior de Investigaciones Científicas-Castalia, 1961.

Libro del caballero Zifar, ed. de Cristina González, México: Rei, 1990.

Luna Mariscal, Karla Xiomara, "El episodio cómico en el Zifar”, en Karla Xiomara Luna Mariscal, Axayácatl Campos y Aurelio González (eds.), Zifar y sus libros: 500 años, México: El Colegio de México, 2015, 125-170.

MalaXecheverría, Ignacio, Bestiario medieval, Madrid: Siruela, 1989.

Thompson, Sтітн, El cuento folklórico, trad. de Angelina Lemmo, Caracas: Universidad Central de Venezuela, 1972. 